\title{
UNIFORM LOCAL LIPSCHITZ CONTINUITY OF EIGENVALUES WITH RESPECT TO THE POTENTIAL IN $L^{1}[a, b]$
}

\author{
XiAO CHEN AND JiANGANG QI
}

Abstract. The present paper shows that the eigenvalue sequence $\left\{\lambda_{n}(q)\right\}_{n \geqslant 1}$ of regular SturmLiouville eigenvalue problem with certain monotonic weights is uniformly Lipschitz continuous with respect to the potential $q$ on any bounded subset of $L^{1}([a, b], \mathbb{R})$.

Mathematics subject classification (2010): 34B05, 45J05, 34L15.

Keywords and phrases: Sturm-Liouville problem, eigenvalue, uniform local Lipschitz continuity.

\section{REFERENCES}

[1] J. H. BarRett, A Prüfer transformation for matrix differential equations, Proc. Amer. Math. Soc., 8(1957) 510-518.

[2] J. Chu, G. Meng And M. Zhang, Continuity and minimization of spectrum related with the periodic Camassa-Holm equation, J. Diff. Equ., 265(4) (2018) 1678-1695.

[3] Q. Kong And A. Zettl, Eigenvalues of regular Sturm-Liouville problem, J. Diff. Equ., 131 (1996) $1-19$.

[4] G. Meng And M. Zhang, Measure differential equations II: Continuity of eigenvalues in measures with weak ${ }^{*}$ topology, technical report, 2009.

[5] G. Meng And M. Zhang, Continuity in weak topology: First order linear syetems of ODE, Acta China Sinica Engl. Ser., 26 (2010) 1287-1298.

[6] M. Moeller And A. Zettl, Differentiable dependence of eigenvalues of operators in Banach spaces, J. Operator. Theory, 36 (1996) 335-355.

[7] B. G. Pachpatte, Inequalities for differential and integral equations, Academic Press, San Diego, 1998.

[8] J. Pöschel And E. Trubowitz, Inverse spectral theory, Pure. Appl Math., vol. 130, Academic Press, New York, 1987.

[9] A. Zettl, Sturm-Liouville theory, Math. Surveys Monogr., vol. 121, Amer. Math. Soc., Providence. RI, 2005.

[10] W. T. REID, A Prüfer transformation for differential systems, Pacific J. Math., 8(3) (1958) 575-584.

[11] B. XIE AND J. QI, No-real eigenvalues of indefinite Sturm-Liouville probelm, J. Diff. Equ., 255(1958) 2291-2301.

[12] Z. Wen, M. YANG AND M. ZHANG, Complete continuity of eigen-pairs of weighted Dirichlet eigenvalue problem, Mediterr J. Math., 15:73 (2018).

[13] P. YAN AND M. ZHANG, Continuity in weak topology and extremal problems of eigenvalues of the p-laplacian, Trans. Amer. Math. Soc., 363 (2011) 2003-2028.

[14] M. Zhang, Continuity in weak topology: High order linear syetems of ODE, Sci. China Ser. A, 51 (2008) 1038-1058.

[15] M. ZHANG, A review on strong continuity results of solutions and eigenvalues in infinitely dimensional parameters, technical report, 2014.

[16] M. Zhang, Z. Wen, G. Meng, J. Qi And B. XIE, On the number and complete continuity of weighted eigenvalues of measure differential equations, Diff. Integ. Equ., 31(9-10) (2018) 761-784. 